\title{
LA MIGRACIÓN CUALIFICADA DE LOS PROFESIONALES DE LA SALUD EN PORTUGAL Y ESPAÑA: UNA APROXIMACIÓN GENERAL ${ }^{1}$ SKILLED MIGRATION OF HEALTH PROFESSIONALS IN PORTUGAL AND SPAIN: A GENERAL APPROACH
}

\author{
Erika Masanet \\ ISCTE - Instituto Universitario de Lisboa, \\ CIES-IUL, Lisboa, Portugal. \\ erika.masanet@iscte.pt \\ Recibido: 8/10/2010 \\ Aceptado: 10/12/2010
}

\begin{abstract}
Resumen
Paralelamente al aumento de la demanda de profesionales de la salud en las últimas décadas, se asiste a un incremento de la migración internacional de los profesionales de la salud. España y Portugal también se han inserido en la dinámica de estos movimientos migratorios principalmente como países de destino, aunque uno se ha incorporado de manera más tardía que otro. El artículo propone un acercamiento general al tema de la migración cualificada de personal sanitario en los dos países ibéricos mediante el abordaje de las siguientes cuestiones: aproximación a las tendencias recientes de la migración internacional de profesionales de la salud a nivel mundial, caracterización general de la población de profesionales de la salud extranjeros que residen en ambos países, descripción de los principales marcos teóricos explicativos de las causas de este tipo de migración y retrato de algunos trazos generales relativos a tres grupos de estos inmigrantes cualificados en Portugal.

Palabras clave: migrantes profesionales de la salud, Portugal, España
\end{abstract}

\footnotetext{
${ }^{1}$ Presentado en el Seminario Internacional Migraciones Circulares, el retorno como estrategia de desarrollo en los países de Origen, celebrado en Alicante, del 6 al 8 de octubre de 2010, organizado por el Instituto Interuniversitario de Desarrollo Social y Paz, con la colaboración del Ministerio de Ciencia e Innovación (Ref. CSO2009-07331-E) y de la Conselleria d'Educació de la Generalitat Valenciana (Ref. AORG/2010/124)
} 


\begin{abstract}
:
Parallel to the increasing demand of health professionals in recent decades, we are seeing an increase in international migration of health professionals. Spain and Portugal have also been inserted in the dynamics of these movements primarily as destination countries, although one was incorporated later. The article proposes a general approach to the topic of skilled migration of health workers in the two Iberian countries by addressing the following issues: approach to recent trends in international migration of health professionals worldwide, characterization of the population of foreign health professionals in both countries, description of the main theoretical frameworks of the causes of this migration and portrait of some general characteristics relating to three groups of these skilled immigrants in Portugal.

Keywords: migrant health professionals, Portugal, Spain
\end{abstract}

\title{
INTRODUCCIÓN
}

Diversos estudios e informes alertan sobre la crisis global de los sistemas de salud y del personal sanitario (Clark et al., 2006; OECD, 2007; Stewart et al., 2007; OECD, 2010). En este sentido, uno de los mayores desafíos que enfrentan los sistemas de salud tanto en los países desarrollados como en los países en desarrollo es la escasez de los profesionales sanitarios.

En su informe anual de 2006, la Organización Mundial de la Salud estimaba que en ese año había una escasez de más de 4,3 millones de personal sanitario en todo el mundo. Particularmente, los países pobres se ven más afectados por esta escasez, ya que de los 57 países con niveles de escasez crítica, 36 pertenecían al África Subsahariana (WHO, 2006). Estos países, junto con Asia, América Latina y el Caribe y, en general los países en desarrollo, sufren una escasez crónica de profesionales de salud (Stewart et al., 2007).

Por su parte, en los países desarrollados esta necesidad es resultado de un aumento significativo de la demanda de profesionales provocado por una serie de cambios demográficos y sociales. El envejecimiento de la población ha contribuido a un aumento de la demanda de los servicios sanitarios y de los tratamientos médicos, aunque algunos estudios no han podido confirmar esta relación de causa-efecto (Adel et al., 2004). Algunos estudios apuntan a otros factores estructurales que afectan a los países de la OCDE, como el proceso de envejecimiento del personal sanitario que provocará una disminución de la oferta de profesionales de la salud, el aumento de los ingresos, las nuevas tecnologías médicas y el incremento de la especialización en los servicios de la salud (OECD, 2007; OECD, 2010). La mayor parte de la literatura sobre migración sugiere que esta demanda se incrementará o al menos continuará en los próximos años debido al envejecimiento de la población y a los cambios tecnológicos (Ray et al., 2006; OECD, 2007). 
En este contexto, una de las soluciones de la escasez de personal sanitario a corto plazo es la contratación o reclutamiento de personal sanitario extranjero. Existen otras medidas para hacer frente a este déficit de personal sanitario: a) el aumento del numerus clausus o número de plazas en las Facultades de Medicina, b) la retención de estos profesionales a través de la mejora de sus condiciones laborales y de las posibilidades de carrera profesional, y c) el retraso en la edad de jubilación. Sin embargo, la inmigración laboral parecer ser la solución rápida adoptada por buena parte de los países por sus efectos inmediatos, a pesar de que puede provocar problemas de equidad internacional a largo plazo -por el impacto negativo en los países en desarrollo-, discriminación laboral hacia los médicos extranjeros (Simoens y Hurst, 2006) y desajustes a medio plazo en los países de destino (González y Barber, 2006).

Por tanto, la contratación de profesionales de la salud de los países menos desarrollados constituye una de las principales respuestas o soluciones de los países desarrollados a este déficit. Aunque la mayoría de estos movimientos se producen en esa dirección, no podemos olvidar que también se producen desplazamientos importantes entre los países desarrollados. En definitiva, la demanda de profesionales de la salud ha aumentado en las últimas décadas tanto en los países en desarrollo como en los desarrollados, pero sobre todo constituye un problema para los países en desarrollo por la emigración de sus médicos y enfermeros hacia los países desarrollados.

La escasez de los profesionales sanitarios también es una realidad en Portugal y en España. En el país luso, la escasez de estos profesionales constituye un problema estructural: el número y la proporción de los profesionales de la salud extranjeros se han incrementado de manera espectacular en las últimas décadas ${ }^{2}$ y no parece que en los próximos años esta tendencia cambie sustancialmente.

Tanto en Portugal como en España los profesionales de la salud extranjeros conforman un segmento importante dentro de la migración altamente cualificada. En el conjunto de países de la OCDE, Portugal constituye uno de los principales destinos de la migración de los profesionales de la salud procedentes de otros países (Lowell, 2008). A diferencia de Portugal, en España la llegada de estos profesionales cualificados es relativamente reciente y ha empezado a ser significativa en los últimos años.

Pero además de ser países de atracción de profesionales sanitarios, también constituyen países de expulsión de este personal cualificado, sobre todo

${ }^{2}$ De acuerdo con el informe de la OCDE (2007), la tasa media de crecimiento anual entre 1975 y 2005 de los médicos formados en el exterior que trabajaban en Portugal fue de cerca un $10 \%$, una de las más elevadas entre los países de la OCDE, junto con Finlandia, Países bajos y Francia. 
España. Muchos médicos y enfermeros españoles han emigrado en los últimos diez años a países como Reino Unido, Portugal o Francia.

El objetivo de este artículo es proporcionar un panorama general de la migración cualificada de personal sanitario en España y Portugal. Para ello se ofrece, en primer lugar, una breve aproximación al escenario mundial reciente de la migración internacional de profesionales de la salud y sus principales tendencias. En segundo lugar, se analizan las estadísticas disponibles en Portugal y España sobre esta población con el fin de proporcionar una caracterización de los profesionales de la salud extranjeros que residen en ambos países. A continuación, se describen sucintamente los principales paradigmas teóricos que nos servirán de apoyo para la explicación de las causas de este tipo de migración. Y, por último, se proporcionarán algunos rasgos generales relativos a tres grupos de profesionales sanitarios extranjeros en Portugal: el grupo de españoles, el grupo de europeos del Este y el grupo de profesionales que han llegado a través de acuerdos bilaterales procedentes de Uruguay y Cuba.

\section{TENDENCIAS GENERALES DE LA MIGRACIÓN DE LOS PROFESIONALES DE LA SALUD EN EL ESCENARIO MUNDIAL}

El incremento de los flujos y la diversificación de los países de origen constituyen las dos principales tendencias recientes en la migración de los profesionales de la salud identificadas por la OCDE (2007). Así, en las últimas dos décadas se asiste un aumento significativo de la migración del personal sanitario en la mayoría de los países de la OCDE, destacando el incremento experimentado en los países europeos (OECD, 2007).

Una de las explicaciones del importante incremento de este flujo migratorio reside en el hecho de que esta migración ha sido usada como una solución rápida a la escasez de personal sanitario en los países de la OCDE (OECD, 2010), tal y como hemos señalado en la introducción. Además de este factor, otros elementos determinantes de este aumento son los cambios en las políticas migratorias para la migración cualificada en general en los países de destino y la combinación de factores como la facilidad en el acceso a la información, la disminución de los costes de viaje y el deterioro de las condiciones laborales en los países de origen (OECD, 2007).

Asimismo, la diversificación de los países de origen es una de las características recientes de estos flujos de migración. A pesar de que los principales países de origen de esta migración continúan siendo Filipinas y la India, recientemente se ha producido un incremento de los flujos en algunos países africanos y en los países de Europa del Este y Central (OECD, 2007).

Antes de pasar a detallar los países de origen y de destino de los profesionales de la salud migrantes, es importante señalar que la mayoría de esta 
movilidad se produce desde los países en desarrollo hacia los países desarrollados, aunque algunos flujos migratorios de profesionales de la salud tienen lugar entre los países desarrollados.

\section{Países de destino}

En general, aquellos países que tienen más inmigración, y particularmente los que atraen más migración cualificada, tienden a albergar un mayor número de profesionales de la salud migrantes (OCDE, 2010). Tal y como afirman Stewart et al. (2007), los países de habla inglesa constituyen los principales destinos tanto de los médicos extranjeros como de los enfermeros. En primer lugar, pasaremos a analizar los datos relativos a los médicos inmigrantes.

Estados Unidos constituye el principal país receptor de médicos extranjeros, además de ser el único país del mundo receptor neto de médicos y enfermeros nacidos en el extranjero (OECD, 2007). Este país, junto con Australia, Canadá y, principalmente, Suiza han experimentado importantes crecimientos de médicos extranjeros en los últimos años. De todos modos, en la mayoría de los países de la OCDE el porcentaje de médicos formados en el extranjero se ha incrementado en los últimos años. Por otro lado, en el Reino Unido el número de médicos formados en el extranjero ha ido disminuyendo desde el año 2003. Con todo, este país aún continua siendo el segundo país de destino de los médicos formados en el exterior, a continuación de los Estados Unidos (OECD, 2010).

El Gráfico 1 refleja la inmigración de los profesionales de la salud en algunos países de la OCDE en el año 2008 (o el último año disponible) a partir de dos tipos de datos en función de la disponibilidad estadística de cada país: el porcentaje de médicos formados en el extranjero sobre el total de médicos en ese país o la proporción de médicos extranjeros sobre el total ${ }^{3}$. En el gráfico podemos observar que, en 2008, Nueva Zelanda, Irlanda y Reino Unido constituían los países con los porcentajes más elevados de médicos formados en el extranjero, 39\%, 36\% y 32\% respectivamente. Del mismo modo, Estados Unidos (26\%) y Australia (23\%) en 2007, y Suiza en 2008 (23\%) también son países con importantes proporciones de médicos formados en el extranjero. En cuanto al porcentaje de médicos extranjeros, en primer lugar se sitúa Noruega con un $16 \%$ sobre el total de médicos en ese país y en segundo lugar Portugal con un $11 \%$.

\footnotetext{
${ }^{3}$ Según el Informe SOPEMI 2007 (OECD, 2007) existen diferencias importantes, tanto en términos absolutos como porcentuales, entre los profesionales de la salud nacidos en el extranjero y aquellos formados en el extranjero. Esto conlleva dificultades para la comparación internacional de las estadísticas sobre la migración de profesionales de la salud.
} 


\section{GRÁFICO 1}

Porcentaje de médicos formados en el extranjero o de médicos extranjeros, algunos países de la OCDE, 2008 (o el último año disponible)

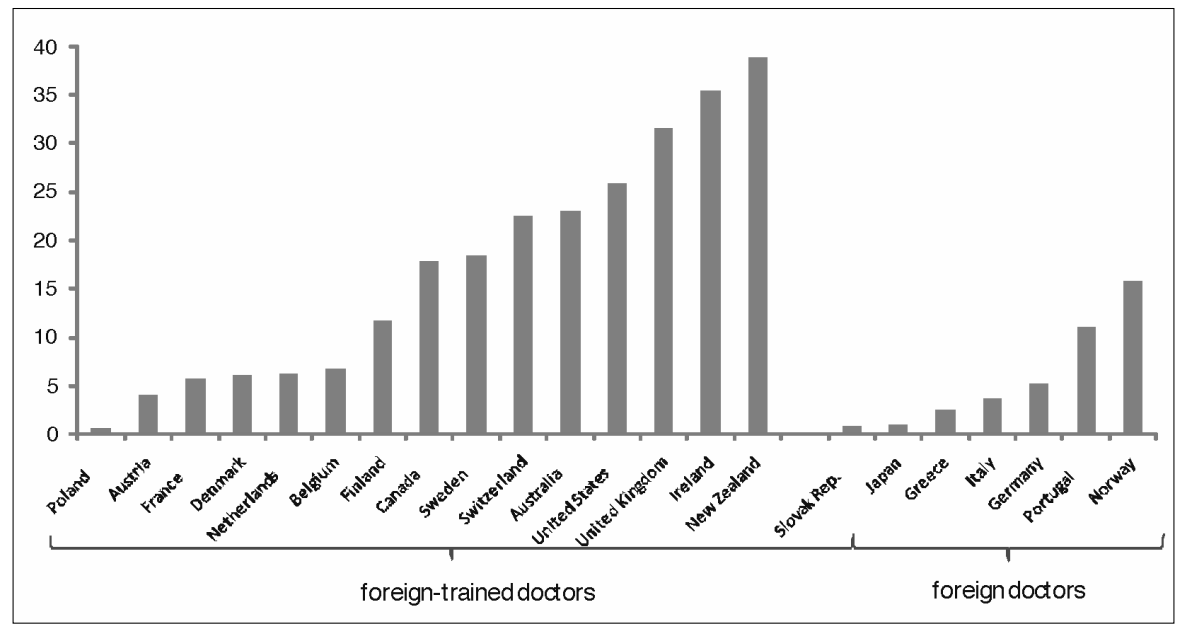

Nota: en los siguientes países el año de referencia es distinto a 2008: Polonia, 2005; Francia, 2005; Países Bajos, 2006; Canadá, 2007; Suecia, 2007; Australia, 2007; Estados Unidos, 2007; República Eslovaca, 2004; Portugal, 2004.

Fuente: OECD (www.oecd.org/health/workforce)

Al igual que sucede con los médicos extranjeros, la migración de enfermeros se ha incrementado en buena parte de los países de la OCDE desde el año 2000, principalmente en Australia, Canadá y Estados Unidos. Contrariamente a esta tendencia, en el Reino Unido e Irlanda la llegada de enfermeros formados en el exterior ha disminuido durante el periodo 2001-2008 (OECD, 2010).

Como se observa en el Gráfico 2, en general, la inmigración de enfermeros tiende a ser menor que la de los médicos en la mayoría de los países de la OCDE analizados, a excepción de Irlanda donde hay un $47 \%$ de enfermeros formados en el extranjero frente al 36\% de médicos formados en el extranjero. A continuación de Irlanda, se sitúan Nueva Zelanda y Australia como los países que albergan mayor porcentaje de enfermeros formados en el exterior entre los países de la OCDE, con un 22\% y $16 \%$ respectivamente.

Países de origen

Tal y como señala el informe SOPEMI 2007 de la OCDE (2007), la distribución de la migración de profesionales sanitarios por regiones de origen refle- 


\section{GRÁFICO 2}

Porcentaje de enfermeros formados en el extranjero o de enfermeros extranjeros, algunos países de la OCDE, 2008 (o el último año disponible)

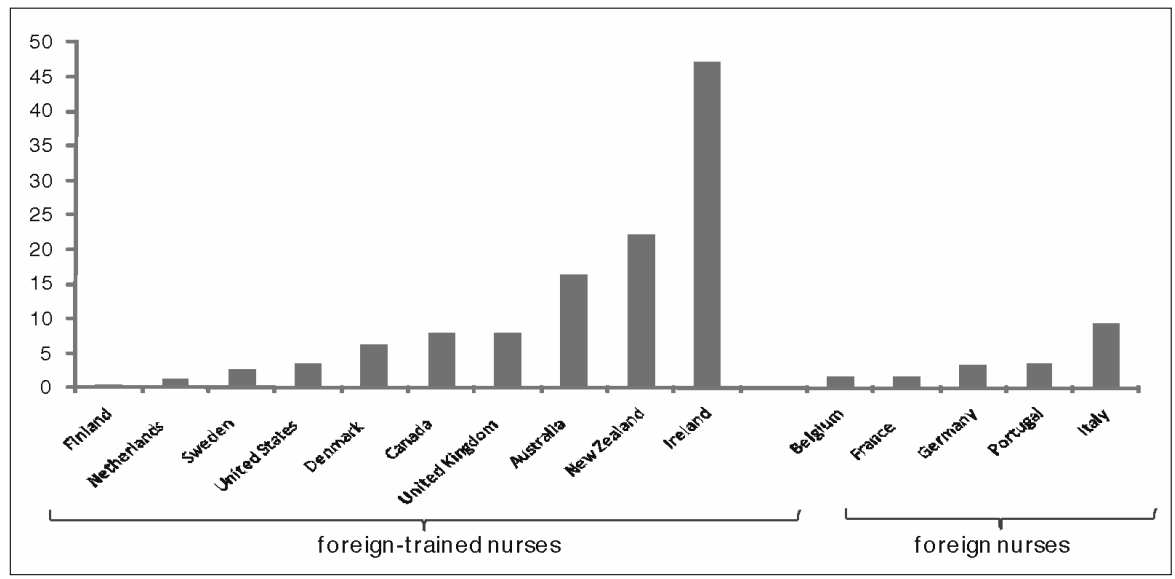

Nota: en los siguientes países el año de referencia es distinto a 2008: Francia, 2005; Australia, 2007; Reino Unido, 2001; Canadá, 2007; Dinamarca, 2005; Estados Unidos, 2004; Suecia, 2007; Países Bajos, 2005.

Fuente: OECD (www.oecd.org/health/workforce)

ja los patrones de la migración en general y, además, se encuentra determinada por factores como el idioma, la proximidad geográfica y cultural, los lazos históricos y políticos y las políticas de migración bilaterales.

Filipinas es el país con mayor número de enfermeras que emigran, mientras que la India es uno de los mayores países exportadores de médicos. Asimismo, los países del Caribe y algunos países africanos, en particular los países de lengua oficial portuguesa y francesa, presentan unas elevadas tasas de emigración de médicos. La situación del sistema de salud en algunos de estos últimos países es preocupante debido a que, además de las elevadas tasas de emigración, presentan una baja densidad de médicos. América Latina también es un importante proveedor de profesionales de la salud que se dirigen principalmente a los Estados Unidos y a algunos países europeos, esencialmente a España (OECD, 2007).

China también cuenta con un importante número de profesionales de la salud trabajando en el exterior, aunque esto parece no haber afectado a su tasa de densidad de médicos, al igual que sucede en la India. La OCDE sostiene que probablemente se asistirá a un aumento de la emigración de los profesionales de la salud en China en los próximos años (OECD, 2007). Además de China, Cuba 
y algunos países de Europa del Este y del África Subsahariana también han visto incrementar sus flujos de profesionales de la salud (Stewart et al., 2007).

Por último, cabe señalar que la movilidad de los profesionales de la salud también es importante dentro de los países de la OCDE, como es el caso de los países nórdicos, Suiza, Nueva Zelanda, Reino Unido, Alemania, Portugal y Francia (OECD, 2007).

\section{CARACTERIZACIÓN DE LOS PROFESIONALES SANITARIOS EXTRANJEROS EN PORTUGAL Y ESPAÑA}

\section{Portugal}

En Portugal existen varias fuentes estadísticas sobre profesionales de la salud: el Ministerio de Salud portugués, los distintos colegios profesionales y el Instituto Nacional de Estadística portugués que, a su vez, se basa en los datos de los colegios profesionales. Cada una de estas fuentes presenta una serie de limitaciones y potencialidades ${ }^{4}$.

En este artículo se han utilizado los datos del Ministerio de Salud porque permiten realizar un análisis diacrónico, ya que este organismo ha venido publicando datos estadísticos sobre recursos humanos extranjeros que prestan servicios en el sector público de la salud desde 1994 hasta 2007, y porque además, es una fuente de información rica y amplia por las variables que utiliza, a saber, país de origen, edad, sexo, grupo de personal, régimen jurídico de empleo y región de salud.

Los datos analizados durante el periodo comprendido entre 1999 y 2007 (Gráfico 3) indican dos etapas en la evolución de los recursos humanos extranjeros: una primera fase de crecimiento desde 1999 hasta 2004, alcanzando el mayor pico en dicho año con 4.490 efectivos, y una segunda etapa caracterizada por una disminución de los profesionales extranjeros en los últimos tres años analizados (2005-2007).

En cuanto a la distribución por sexo, la tasa de feminización en el año 2007 fue del $61 \%$, de modo que las mujeres predominan entre los profesionales de la salud extranjeros, sobre todo en el área de enfermería. Por otro lado, la evolución de la estructura etaria constata un progresivo envejecimiento de los profesionales extranjeros.

Por lo que respecta a los países de origen, hasta 1998 los profesionales extranjeros procedían mayoritariamente de las PALOP (Países Africanos de Lengua Oficial Portuguesa) (un $45 \%$ ) y a partir de esa fecha se produce un

\footnotetext{
${ }^{4}$ Para conocer las ventajas e inconvenientes de cada fuente, consultar Baganha y Ribeiro (2007).
} 


\section{GRÁFICO 3}

Evolución de los Recursos Humanos Extranjeros en el Ministerio de Salud de Portugal, 1999-2007

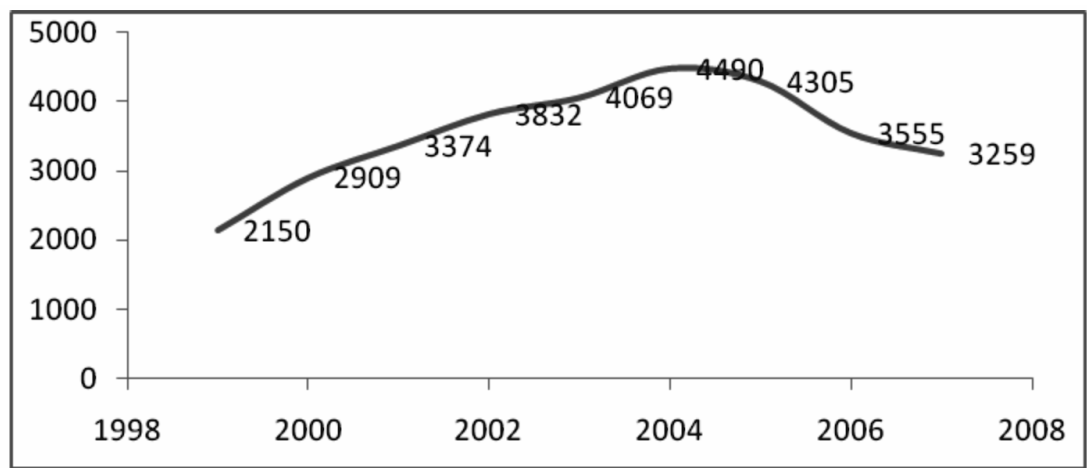

Fuente: elaboración propia a partir de la Administração Central do Sistema de Saúde, Ministerio de Salud de Portugal.

cambio, ya que en 1999 los profesionales de la Unión Europea (53\%), en su mayoría españoles y españolas, adquieren una mayor importancia.

Según datos correspondientes a 2007, los principales países de procedencia son, en primer lugar, España (con 1.356 efectivos), dentro de los países europeos, Angola (376), en el conjunto de los países africanos de lengua oficial portuguesa (PALOP), Brasil (363), y dentro de la agrupación de Otros Países, cabe destacar Ucrania, Moldavia y Rusia (Gráfico 4).

\section{GRÁFICO 4}

Principales países de origen de los Recursos Humanos Extranjeros, 2007

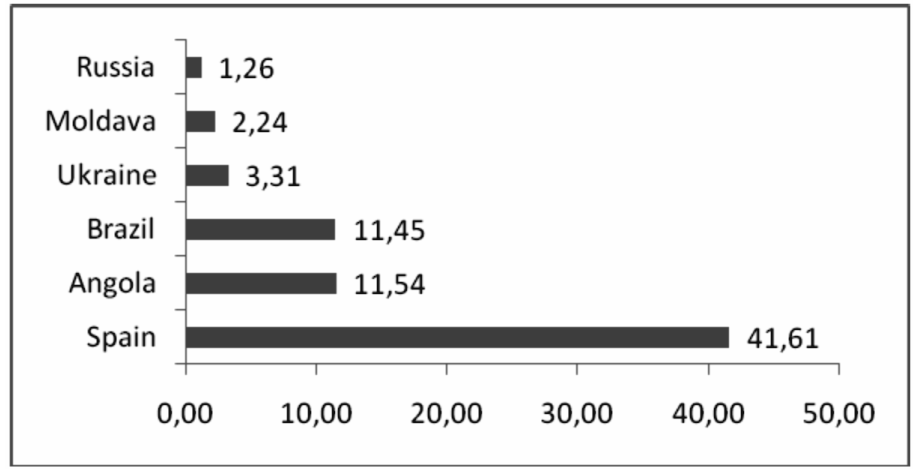

Fuente: elaboración propia a partir de la Administração Central do Sistema de Saúde, Ministerio de Salud de Portugal. 
A pesar del predominio de los profesionales españoles, se observa una disminución de éstos a partir de 2005, sobre todo debido al flujo de retorno de enfermeros y enfermeras españolas. Al mismo tiempo que se produce esta reducción del número de profesionales españoles, también tiene lugar una mayor diversificación de los países de origen por el crecimiento de los profesionales procedentes de los países de Europa del Este: Ucrania, Rusia, Rumanía y Moldavia.

El Servicio Nacional de Salud de Portugal se organiza en 5 regiones de salud: Norte, Centro, Lisboa e Vale do Tejo, Alentejo y Algarve. Las estadísticas correspondientes a 2007 indican una mayor presencia de los profesionales extranjeros en la Región de Lisboa y Vale do Tejo (1.294) y en la región Norte (942).

Analizando por país de origen según región, tenemos que los profesionales extranjeros procedentes de la UE y, dentro de este grupo los y las españolas, se concentraban en 2007 en la regiones del Norte, Lisboa e Vale do Tejo y Algarve. Por otro lado, los profesionales oriundos de las PALOP ejercían mayoritariamente en las regiones de Lisboa e Vale do Tejo, Norte y Centro, de manera similar a los profesiones de Otros Países, mientras que los profesionales brasileños se encontraban concentrados en el Norte y en Lisboa y Vale do Tejo.

La distribución de los recursos humanos extranjeros por grupo profesional con datos de 2007 muestra que los grupos más numerosos son los médicos (1.903) enfermeros (836) y auxiliares (259). Los dos primeros grupos (médicos y enfermeros) representan el $84 \%$ del total de los profesionales extranjeros en 2007.

Si analizamos con más detalle estos dos grupos, vemos que España es el principal país de origen de los médicos y enfermeros extranjeros que ejercen sus funciones en el Ministerio de la Salud portugués. Mientras que los médicos españoles se concentran principalmente en la Región Norte del país, los enfermeros españoles tienen una mayor presencia en la Región de Lisboa y Vale do Tejo.

A diferencia de los médicos españoles, el personal de enfermería español ha ido disminuyendo en los últimos años analizados, sobre todo a partir de 2005, pasando de tener 1.271 efectivos en 2001 a 431 en 2007.

\section{España}

En España no se sabe con exactitud el número de profesionales sanitarios extranjeros que ejercen en el país. La OMS estima que hay aproximadamente unos 25.000 médicos extranjeros, lo que supondría un 12,5\% del total.

El Ministerio de Sanidad y Política Social no dispone de este tipo de información de manera centralizada. Una aproximación al número de profesiona- 
les extranjeros que trabajan en España puede hacerse a través del número de títulos de Medicina homologados por el Ministerio de Educación para ejercer la profesión en nuestro país, cuyos datos indican una evolución creciente en el número de homologaciones en los últimos años, creciendo a un ritmo de 3.653 médicos extranjeros en los últimos cuatro años.

Otra fuente de datos sobre los profesionales sanitarios extranjeros en España son los colegios profesionales que son los que vamos a presentar a continuación, concretamente los datos proporcionados por el Colegio Oficial de Enfermería de Madrid y el Colegio Oficial de Médicos de Barcelona. Aunque estos datos, evidentemente, no se pueden generalizar al resto del país, sí que nos pueden dar algunas pistas o indicaciones acerca de las tendencias existentes en el territorio español.

Según las estadísticas del Colegio de Enfermería de Madrid correspondientes a los datos acumulados hasta agosto de 2010 (Gráfico 5), Perú (32,89\%), Rumanía (13,41\%), Colombia (12,56\%) y Ecuador (5,32\%) constituyen los principales países de origen de los enfermeros y enfermeras colegiadas en la Comunidad de Madrid. Analizando por grupos de países, se detecta una mayor presencia de enfermeras y enfermeros procedentes de América Latina.

Pasando a los datos sobre médicos extranjeros, el Informe Anual 2009 del Colegio de Médicos de Barcelona señala que, uno de los fenómenos que afectan

\section{GRÁFICO 5}

Principales países de origen de las enfermeras colegiadas en Madrid. Datos acumulados hasta agosto 2010

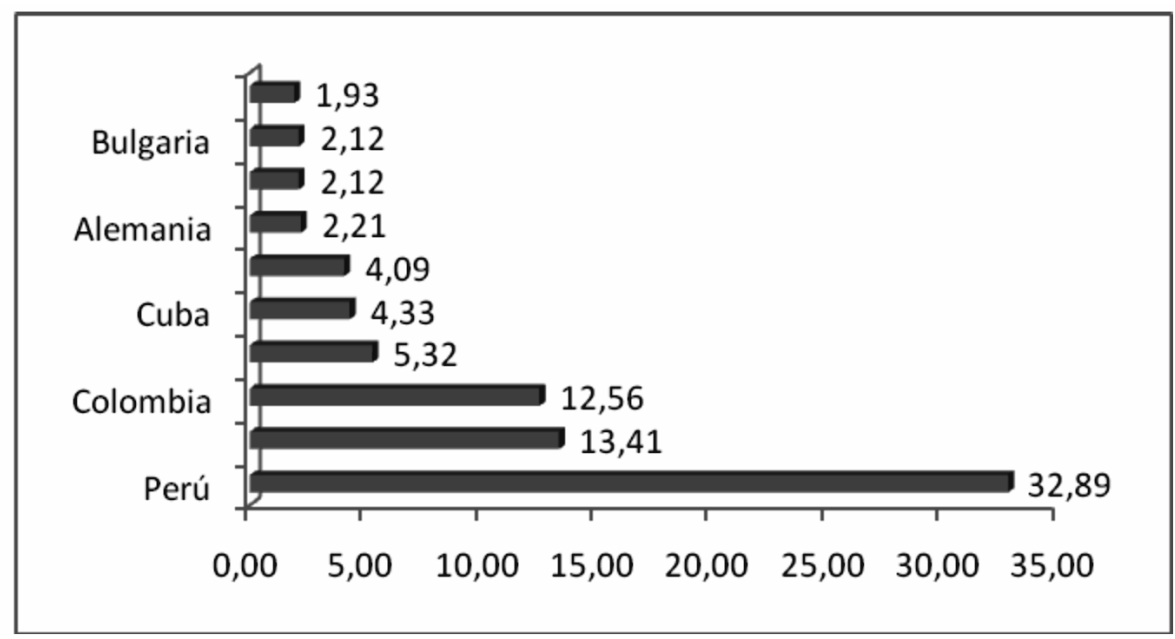

Fuente: elaboración propia a partir del Colegio Oficial de Enfermería de Madrid 
a la demografía colegial, además del proceso de envejecimiento y feminización, es la llegada de médicos extranjeros (Colegio de Médicos de Barcelona, 2009).

Durante el año 2008 y 2009, el grupo de extranjeros fue el más numeroso entre las nuevas colegiaciones (el 57,8\% de los nuevos colegiados en 2009 son de origen extranjero). Asimismo, también es de destacar el crecimiento de médicos extranjeros entre los nuevos colegiados MIR ${ }^{5}$ durante 2009 (el 44,9\% de los nuevos colegiados MIR de 2009 son de origen extranjero).

Como se observa en el Cuadro 1, el 15,6\% del total de médicos colegiados son de origen extranjero y entre estos, la mayoría proceden de América Latina (que representan el 75,9\% de los extranjeros), seguido a bastante distancia de los nacidos en la UE15 (9,7\%) y los oriundos de Europa del Este (5,2\%).

\section{CUADRO 1}

Distribución de los médicos colegiados en Barcelona según procedencia, 2009

\begin{tabular}{l|c|c|c}
\hline Lugar de nacimiento & Números & $\%$ & $\%$ médicos extranjeros \\
\hline Cataluña & 19.356 & 65,5 & \\
\hline Resto de España & 5.567 & 18,8 & \\
\hline Total extranjeros & 4.616 & 15,6 & \\
\hline América Latina y el Caribe & 3.502 & 11,9 & 75,9 \\
\hline UE15+EEE & 449 & 1,5 & 9,7 \\
\hline Oriente Medio & 219 & 0,7 & 4,7 \\
\hline África & 123 & 0,4 & 2,6 \\
\hline Europa del Este & 242 & 0,8 & 5,2 \\
\hline Otros & 81 & 0,3 & 1,7 \\
\hline Total & 29.539 & 100,0 & 100,0 \\
\hline
\end{tabular}

Fuente: Colegio de Médicos de Barcelona

En cuanto a la evolución entre 2000 y 2009, es de resaltar la importante variación porcentual experimentada por el grupo de extranjeros colegiados, que es del 206,7\%. Por grupos de países, cabe destacar el crecimiento de los nacidos

${ }^{5}$ Médico Interno Residente, es decir, el médico que se está formando como especialista. Se trata de un programa de formación especializada para la adquisición de las habilidades, capacidades y responsabilidades necesarias para la especialización y que se realiza en aquellos centros debidamente acreditados por el Ministerio de Sanidad de España. La duración del programa de formación suele oscilar entre cuatro y cinco años. La forma de acceder a este programa de formación se realiza a través de un concurso-oposición, conocido como examen MIR. 
en América Latina (270,6\%) y el rápido crecimiento de los nacidos en Europa del Este (476\%), aunque este último se trata de un colectivo pequeño en términos absolutos.

En cuanto a los países de origen de los nuevos colegiados extranjeros (Cuadro 2), cuatro países (Colombia, Argentina, Perú y Venezuela) agrupan a más de la mitad de las nuevas incorporaciones de médicos extranjeros en 2009.

Por tanto, de la comparación en la composición de los profesionales de la salud extranjeros en España y Portugal, extraemos que en Portugal hay una mayor presencia de médicos procedentes de la Unión Europea, conformados en su mayoría por profesionales españoles, mientras que en España se evidencia un predominio de los profesionales procedentes de América Latina, aunque sin una presencia destacada de un país determinado. Por otro lado, un fenómeno similar que se detecta en ambos países es el importante crecimiento de los profesionales sanitarios procedentes de Europa del Este en los últimos años.

\section{CUADRO 2}

Ranking de los 10 primeros países de origen de los nuevos colegiados extranjeros en Barcelona, 2009

\begin{tabular}{l|c|c}
\hline País de nacimiento & Números & \% de médicos extranjeros \\
\hline Colombia & 121 & 15,5 \\
\hline Argentina & 103 & 13,2 \\
\hline Perú & 93 & 11,9 \\
\hline Venezuela & 82 & 10,5 \\
\hline México & 50 & 6,4 \\
\hline Cuba & 41 & 5,2 \\
\hline Bolivia & 38 & 4,9 \\
\hline Rep. Dominicana & 33 & 4,2 \\
\hline Italia & 32 & 4,1 \\
\hline Paraguay & 27 & 3,5 \\
\hline
\end{tabular}

Fuente: Colegio de Médicos de Barcelona

\section{MARCOS EXPLICATIVOS SOBRE LAS CAUSAS DE LA MIGRACIÓN INTERNACIONAL DE LOS PROFESIONALES SANITARIOS}

La naturaleza dinámica, multidimensional y compleja del fenómeno migratorio, hace necesario abordar su estudio desde la complementariedad de diferentes aproximaciones teóricas. Al igual que el fenómeno migratorio en general, para la comprensión de la migración cualificada de los profesionales de la 
salud es fundamental la utilización de diversos paradigmas teóricos con el fin de abarcar la totalidad de los factores que influyen en este tipo de migración (de carácter económico, político, social y cultural) y los distintos niveles de análisis que intervienen (macro, meso y micro).

A pesar de esta premisa general, la mayor parte de la literatura sobre la migración cualificada en general, y la migración cualificada de profesionales de la salud en particular, se basa en el enfoque teórico de los factores de atracción y de expulsión (push and pull factors) para explicar los determinantes de esta migración. Además de fundamentarnos en esta teoría, en este apartado presentaremos de manera sucinta otros enfoques teóricos que nos ayudarán a explicar y comprender las causas de este tipo de migración.

\section{El modelo de atracción-expulsión (Push and pull factors)}

En general, los factores de expulsión y atracción de la migración cualificada, y también de los migrantes en general, coinciden con los determinantes que impulsan la migración de los profesionales de la salud. De todos modos, el Cuadro 3 ilustra, de manera específica, el conjunto de factores de atracción y expulsión que intervienen en la migración de los profesionales de la salud.

Como se aprecia en el Cuadro 3, las causas en los países de origen o factores de expulsión comprenden condiciones de naturaleza laboral y profesional: bajos salarios, condiciones laborales precarias e inestables, riesgos relacionados con el trabajo y escasas posibilidades de desarrollo profesional. Los problemas socioeconómicos, como la baja calidad de vida y la carencia de oportunidades (fundamentalmente educacionales) para los hijos, también son elementos influyentes en la emigración de estos profesionales. Además, en determinados países, los factores de carácter político (violencia, guerras e inestabilidad política) también actúan como determinantes para la emigración de los profesionales de la salud. Tal y como apunta Stewart et al. (2007), estos factores de expulsión normalmente están presentes en los países en desarrollo, aunque también pueden encontrarse en algunos países desarrollados, concretamente los factores relacionados con las condiciones laborales y profesionales, originando la emigración del personal de la salud desde un país desarrollado a otro país desarrollado.

Por su parte, las condiciones de los países de destino que motivan la emigración o pull factors de los profesionales de la salud están relacionadas con las mejores oportunidades laborales y profesionales que ofrece el país (mejores salarios y condiciones laborales, mayores posibilidades de promoción en la carrera profesional y ambiente de trabajo más seguro), así como también a factores de corte político (estabilidad política, carácter democrático de las sociedades de destino y facilidades a este tipo de migración) y de bienestar social (mejor calidad de vida y posibilidad de ofrecer un mejor futuro a los hijos). 


\section{CUADRO 3}

Factores de atracción y expulsión en la migración del personal sanitario

\begin{tabular}{|c|c|}
\hline Push factors & Pull factors \\
\hline - Inadecuada compensación & - Mejor compensación \\
\hline $\begin{array}{l}\text { - Precarias condiciones laborales/ } \\
\text { insatisfacción laboral }\end{array}$ & $\begin{array}{l}\text { - Mejores condiciones laborales/ } \\
\text { mayor satisfacción laboral }\end{array}$ \\
\hline $\begin{array}{l}\text { - Riesgos relacionados con el trabajo } \\
\text { (VIH, SIDA, tuberculosis, etc.) }\end{array}$ & - Ambiente de trabajo más seguro \\
\hline $\begin{array}{l}\text { - Falta de oportunidades en el } \\
\text { desarrollo profesional }\end{array}$ & $\begin{array}{l}\text { - Mayores oportunidades de } \\
\text { promoción profesional }\end{array}$ \\
\hline - Mala calidad de vida & - Mejor calidad de vida \\
\hline $\begin{array}{l}\text { - Inestabilidad política/guerra/ } \\
\text { luchas étnicas, etc. }\end{array}$ & - Estabilidad política \\
\hline $\begin{array}{l}\text { - Carencia de oportunidades } \\
\text { (educacionales, etc.) para los niños }\end{array}$ & $\begin{array}{l}\text { - Mejores oportunidades } \\
\text { para los niños }\end{array}$ \\
\hline
\end{tabular}

Fuente: Kline, 2003

Los factores de atracción que están presentes en los países desarrollados ejercen una mayor influencia en los profesionales que viven en los países en desarrollo que en quienes residen en los países desarrollados, aunque también impulsan la migración desde un país desarrollado a otro país desarrollado, al igual que los factores de expulsión (Stewart et al., 2007). En los países desarrollados, los migrantes profesionales de la salud suelen encontrar mejores condiciones laborales y estilos de vida, así como oportunidades para el desarrollo personal y profesional que no serían posibles en sus países de origen (Buchan y Dovlo, 2004). Sin embargo, en muchas ocasiones las condiciones laborales de estos migrantes suelen ser más desfavorables que la de los profesionales autóctonos. Una de las explicaciones de estas diferencias en las condiciones laborales estaría relacionada con las dificultades para el reconocimiento de sus cualificaciones y experiencia y la consecuente necesidad de someterse a programas de reciclaje (OECD, 2007).

\section{Las teorías neocoloniales y la importancia de los lazos históricos}

Este conjunto de teorías se centra en las características del sistema de explotación que marcarían las relaciones económicas entre las antiguas potenciales coloniales y las ex-colonias. Bajo este paradigma teórico, el neocolonialismo es percibido como una forma de imperialismo, en el cual la transferencia de 
recursos se produce a través de la creación de lazos de dependencia económica y cultural con las antiguas colonias (Clark et al., 2006).

Aplicado al caso de los migrantes profesionales de la salud, la escasez de estos profesionales en las antiguas metrópolis puede ser paliada a través del incentivo a la migración de los profesionales de la salud desde las antiguas colonias hacia estos países. Este mecanismo de movilidad puede ser más efectivo cuando la antigua metrópoli se involucra activamente en la formación educativa de los lugares de origen. Por tanto, esta perspectiva teórica percibe la migración de los profesionales de la salud como una de las manifestaciones del sistema neocolonial de explotación: la migración de los profesionales de la salud a menudo responde a los intereses de las antiguas potenciales coloniales y, en algunos casos, el tipo de formación recibida en las ex -colonias se adapta a las necesidades de los países de destino o antiguas potenciales coloniales (Clark et al., 2006).

Prueba de ello es que una gran parte de los emigrantes profesionales de la salud se desplazan hacia la antigua metrópoli de su país. Es el caso del importante número de profesionales de la salud que trabajan en Portugal procedentes de las ex -colonias portuguesas como Angola, Mozambique o Cabo Verde. De igual modo, la mayoría de los enfermeros y médicos inmigrantes en el Reino Unido han emigrado desde países que formaban parte del Imperio Británico, a excepción de los procedentes de Filipinas (Stewart et al., 2006).

Por otro lado, las vinculaciones culturales e históricas creadas durante el periodo de colonización también ejercen una influencia notable en el origen y destino de estos flujos migratorios, fundamentalmente la proximidad cultural e idiomática. Autores como Sayad (1998) y Sassen (1988) afirman cómo los flujos migratorios actuales entre países responden muchas veces a la existencia de vinculaciones históricas previas, y la migración cualificada de profesionales de la salud no se escapa a esta influencia. Así, el flujo migratorio de profesionales de la salud desde América Latina hacia España es un claro ejemplo de las conexiones históricas previas entre países emisores y receptoras, basadas en la historia de colonización y la influencia cultural española. Y del mismo modo se puede aplicar a los casos de Portugal y Reino Unido mencionados en el párrafo anterior.

\section{La teoría de las redes de migración}

Las redes de migración pueden definirse como el conjunto de relaciones interpersonales que vinculan a los migrantes con los familiares, amigos o conciudadanos que permanecen en la sociedad de origen. Estas redes proporcionan información, ayuda económica y laboral, alojamiento, apoyo afectivo, etc. En este sentido, las redes constituyen el canal a través del cual circulan 
bienes intangibles como las informaciones, los apoyos, las ideas, las experiencias, etc., así como también las remesas monetarias y la ayuda financiera necesaria para emprender el viaje. De este modo, al reducir los costes y riesgos del desplazamiento, las redes facilitan la inserción del migrante y, en definitiva, incentivan la migración (Massey et al., 1998).

Las abundantes investigaciones realizadas sobre migraciones y redes sociales coinciden en la importancia de éstas en la decisión de emigrar y en la elección del país de destino, además de constituir uno de los principales factores que determinan la dirección de los flujos migratorios, el carácter selectivo y la intensidad de los movimientos migratorios.

Además, constituye una herramienta teórica especialmente útil para explicar el mantenimiento de la migración que estaría relacionado con la creación y el establecimiento de redes sociales en el país de destino que proporcionan información sobre las oportunidades de empleo y diversos tipos de apoyo una vez llegan a la sociedad de destino. La potencialidad de este enfoque teórico a la hora de explicar los flujos migratorios es perfectamente aplicable al caso de la migración cualificada de los profesionales de la salud. Como veremos en el siguiente apartado, las redes sociales juegan un papel importante en los factores explicativos de la emigración de los profesionales de la salud españoles hacia Portugal.

\section{La relevancia del factor político en la migración cualificada de personal sanitario}

La dimensión política adquiere una especial relevancia en la migración cualificada de profesionales de la salud, ya que en ocasiones esta migración responde a políticas activas de reclutamiento por parte de los Estados, empleadores o agencias. En este contexto, las instituciones estatales y los acuerdos bilaterales o internacionales juegan un papel relevante en la dirección de estos flujos migratorios, su desarrollo y las condiciones en que se produce la inserción de estos migrantes en los países de destino, limitando así, tal y como sostiene Stewart et al. (2007), el poder del libre mercado y de las decisiones individuales. Incluso en algunos países de destino, el Estado es el principal agente de reclutamiento de los migrantes internacionales profesionales de la salud.

Los acuerdos bilaterales o multilaterales entre países o instituciones de salud para la gestión de esta migración constituyen instrumentos para la regulación de la migración de los profesionales de salud que, en general, establecen límites en el número de profesionales que pueden ser contratados, con el fin de reducir el impacto negativo en los sistemas de salud de los países de origen.

Sin embargo, son pocos los países que han adoptado acuerdos bilaterales para el reclutamiento internacional de profesionales de la salud, de modo 
que, como afirma la OCDE (2007: 163): "Bilateral agreements do not play an important role so far". En Europa, el Reino Unido es el único país que ha recurrido de manera frecuente a acuerdos bilaterales y memorandos de entendimiento con países no pertenecientes a la OCDE para la contratación internacional de personal médico y de enfermería. Como ejemplos, ha firmado un memorando de entendimiento con India (2002), un acuerdo con Sudáfrica para el intercambio recíproco de educación en salud y de personal (2003) y un Protocolo de Cooperación para el reclutamiento de profesionales de la salud con China (2005) (OECD, 2007). En el contexto europeo, Reino Unido firmó en el año 2000 un acuerdo con España para la contratación estructurada de enfermeras españolas para el Sistema Nacional de Salud Británico. Este acuerdo explicaría, en parte, el importante flujo migratorio de enfermeras españolas al Reino Unido en los primeros años del siglo XXI.

Asimismo, el Reino Unido posee uno de los programas de reclutamiento más sistemáticos y coordinados del mundo llevado a cabo por el Sistema Nacional de Salud Británico (SNSB), basado en diversas estrategias de reclutamiento según el tipo de profesional de que se trate. El SNSB normalmente recluta a personal médico de forma individual, mientras que el de enfermería suele ser contratado en grupos de 10, 20 o más personas procedentes de un determinado país. Además de ser un agente de reclutamiento, el SNSB también proporciona información sobre los lugares de trabajo, el alojamiento y los procedimientos administrativos de inmigración (Buchan y Dovlo, 2004; Buchan, Jobanputra y Gough, 2004).

\section{GRUPOS DE PROFESIONALES SANITARIOS EXTRANJEROS EN PORTUGAL: ESPAÑOLES, EUROPEOS DEL ESTE Y LATINOAMERICANOS}

El grupo de españoles

Una de las principales causas de la emigración de los enfermeros y enfermeras españoles es la necesidad de adquisición de experiencia profesional con el fin de acumular puntos que futuramente sean válidos para las bolsas de trabajo en España (Leal, 2003; Baganha y Ribeiro, 2007). Por tanto, estos profesionales perciben la migración como una forma de tener mejores oportunidades de empleo futuras en el país de origen, de modo que este tipo de migración no constituye una real adquisición de capital humano por parte del país (Leal, 2003).

Entre los motivos de la elección de Portugal como país de destino, se encuentran la proximidad del país, las afinidades lingüísticas y culturales, la existencia de empleo en Portugal, la contabilización del tiempo de servicio 
para efectos de inserción en el mercado de salud español y la existencia de redes sociales en el país, es decir, de otros enfermeros españoles (Leal, 2003).

Según el estudio de Baganha y Ribeiro (2007), los procesos de reclutamiento de los enfermeros españoles por parte de las instituciones del sector público portugués se realizan a través de anuncios publicados en la prensa española (regional o local) o bien a través de contactos con las asociaciones profesionales españolas, ubicadas sobre todo en las regiones fronterizas con España (Extremadura, Andalucía y Galicia). Por otro lado, los mecanismos de reclutamiento de las instituciones privadas o de las instituciones públicas con gestión privada son más diversificados: anuncios publicados en la prensa escrita, Internet, anuncios divulgados en hospitales españoles, contacto con profesionales seniores, contactos con las Escuelas de Enfermería y con sindicatos de enfermería españoles y redes informales.

En cuanto al perfil de las enfermeras españolas, se trata de un grupo con una experiencia de trabajo reducida porque en su mayoría son recién formadas o incluso con experiencias de desempleo de corta duración en su país de origen. Hay una elevada rotación de estas enfermeras en los servicios de salud portugueses que se explica por la necesidad de adquirir puntuación que sólo es posible a través de una movilidad institucional. Además, en muchos casos, se produce un proceso de descualificación profesional de las enfermeras españolas porque pasan a realizar tareas propias de la categoría de auxiliar de enfermería (Baganha y Ribeiro, 2007).

Las investigaciones al respecto constatan que la mayoría de estos profesionales regresan a su país de origen y los datos estadísticos así lo confirman. Los factores de retorno de los y las enfermeras españolas son los mejores salarios y las mejores condiciones socio-económicas en España, así como motivos familiares.

Si la partida de este personal profesional se puede considerar una fuga de cerebros o "brain drain" para España, posteriormente se convierte en una migración temporal, transitoria o circular ("brain circulation"), ya que estos profesionales de la salud regresan a su país de origen, llevando consigo nuevas competencias y conocimientos, y convirtiéndose en un beneficio para el país de origen.

En el caso de los médicos españoles, y según el estudio realizado por Baganha y Ribeiro (2007), el motivo principal por el que emigran a Portugal es para realizar el Internato Complementar (formación de especialidad, que es el equivalente al MIR en España), después de que en España no pasaron el examen de acceso a la especialidad (examen MIR). Además, la existencia de redes sociales en Portugal o la experiencia anterior de movilidad cuando eran estudiantes (ERASMUS), contribuyó a la decisión migratoria. Otros factores de atracción específicos del sistema de salud portugués son la mayor seguridad contractual y las oportunidades de inserción laboral en hospitales de excelencia. 


\section{Los médicos y enfermeros de Europa del Este}

El caso de los médicos y enfermeros procedentes de Europa del Este adopta un cariz totalmente diferente al resto de grupos de profesionales extranjeros, ya que constituye una mano de obra que a pesar de haber emigrado por razones laborales no lo hacen con el objetivo de integrarse en la profesión que desempeñaban en su país de origen. Este grupo de inmigrantes se integran laboralmente en el segmento secundario del mercado de trabajo, es decir, en el sector de la construcción, el servicio doméstico y la hostelería, de modo que experimentan procesos de movilidad profesional descendente o descualificación profesional en Portugal.

En comparación con los profesionales formados en un país de la UE en el que el reconocimiento del título es automático, el proceso de reconocimiento académico y profesional de los médicos y enfermeros de Europa del Este es un procedimiento largo, burocrático, caro (por los gastos asociados a las traducciones y legalizaciones de los documentos) e incompatible con el ejercicio de una actividad laboral, ya que exige una dedicación exclusiva en la preparación para los exámenes y evaluaciones requeridos por las facultades.

Ante esta adversa situación y los obstáculos y dificultades para el reconocimiento de las cualificaciones de este grupo de inmigrantes, surgió el proyecto de apoyo a médicos y enfermeros inmigrantes de la Fundación Calouste Gulbenkian y la ONG "Servicio Jesuita a los Refugiados".

Se trata de un programa innovador, atípico e inusual, que tiene como objetivo apoyar el reconocimiento de cualificaciones y la integración social y profesional de inmigrantes altamente cualificados procedentes de países de fuera de la UE, y principalmente se destina a aquellas personas que ya estaban trabajando en el país en otros sectores. Este proyecto fue fruto de la solidaridad y la filantropía de un grupo de personas con la voluntad de cambiar la vida de estos inmigrantes. Por tanto, su origen tuvo un apoyo de la sociedad civil y no estatal o institucional.

Como en Portugal hay una reconocida carencia de médicos, esta área profesional fue la primera seleccionada por el proyecto, bajo el nombre de "Apoio à Profissionalização de Médicos Imigrantes". Este proyecto para médicos se inició en 2002 y concluyó en 2005, y fue totalmente financiado por la Fundación Calouste Gulbenkian. Los principales beneficiarios de este proyecto fueron mayoritariamente los procedentes de países de Europa del Este (95\%) (Valle et al., 2008). El programa abarcó los siguientes tipos de apoyo:

1) Apoyo económico para el pago de gastos de traducción de los documentos, bibliografía recomendada para la preparación de los exámenes y becas de nueve meses para realizar a tiempo completo prácticas en hospitales y centros de salud. 
2) Cursos intensivos de Lengua Portuguesa, incluyendo, la formación de portugués técnico.

3) Seguimiento personalizado de los candidatos en la fase de formación y, más tarde, cuando ya obtienen el reconocimiento de sus cualificaciones y autorización para el ejercicio de la profesión en Portugal, seguimiento y apoyo para su integración profesional, social y familiar.

4) Firma de convenios con el Serviço de Estrangeiros e Fronteiras (SEF) para permitir que los candidatos suspendan sus contratos de trabajo y se dediquen exclusivamente al estudio y a la realización de prácticas sin ser penalizados, y con el Ministerio de la Salud para la inserción laboral de estos profesionales.

Las dos entidades organizadoras del primer proyecto decidieron realizar un segundo en el área de enfermería, que se desarrolló entre 2005 y 2007, bajo el título de "Projecto de Equivalência de cualificaciones Académicas e Profissionais de Enfermeiros Imigrantes". Todos los candidatos de este programa eran oriundos de países de Europa del Este.

En cuanto a los resultados de estos dos proyectos, 107 médicos y 45 enfermeros inmigrantes obtuvieron la equivalencia de sus titulaciones académicas y la autorización para ejercer su profesión en Portugal. Se encuentran actualmente trabajando en el Sistema Nacional de Salud portugués y gracias a estos proyectos pudieron reiniciar sus carreras profesionales que fueron interrumpidas con la emigración. Esto también supone un beneficio para la sociedad portuguesa porque cuentan con la contribución y dedicación de estos profesionales en un área donde hay una continua carencia de personal (Valle et al., 2008).

Debido a los buenos resultados de los dos proyectos, se lanzó en agosto de 2008 un nuevo proyecto de apoyo a médicos inmigrantes titulado "Integração Profissional de Médicos Imigrantes" y que está a punto de ser concluido. El objetivo de este nuevo programa es integrar 150 médicos inmigrantes que se encuentran residiendo legalmente en el país en el Sistema Nacional de Salud. A diferencia de los dos primeros, este programa está totalmente financiado por el Ministerio de Salud portugués.

La contratación a través de acuerdos bilaterales: los médicos uruguayos y cubanos La contratación a través de acuerdos gubernamentales bilaterales son procesos de reclutamiento más estructurados y hay que resaltar la importancia de éstos en el actual contexto de crisis global del personal sanitario para la gestión más ética y equitativa del reclutamiento de los profesionales de la salud. 
La contratación de médicos cubanos y uruguayos han sido las primeras experiencias del gobierno portugués en la formalización de acuerdos bilaterales con los gobiernos de otros países para reclutar profesionales de la salud extranjeros.

En septiembre de 2007, los Ministerios de Salud de Portugal y Uruguay firmaron el Memorando de Entendimiento en materia de salud por el cual se establecían dos líneas principales de actuación: a) Procesos de intercambios o estancias de médicos uruguayos en Portugal durante unos meses en centros de alta especialización y complejidad, concretamente en unidades de trasplantes hepáticos; y b) la contratación por tres años de médicos uruguayos no especialistas para trabajar en los servicios de emergencia médicas de los hospitales portugueses (INEM).

El proceso de selección de los médicos uruguayos para trabajar en el INEM se realizó en Uruguay a través de la realización de una serie de exámenes teóricos y prácticos para evaluar los conocimientos y capacidades de los candidatos a seleccionar. Para el proceso de selección se desplazaron docentes universitarios de distintas Facultades de Medicina en Portugal, así como representantes de la Ordem dos Médicos ("Colegio Profesional de Médicos"). Finalmente pasaron estas pruebas 15 médicos, de los cuales finalmente llegaron 14 a Portugal. Estos médicos recibieron una formación lingüística previa en su país (cursos intensivos de lengua portuguesa). Una vez en Portugal pasaron por un programa de aprendizaje y adaptación al sistema sanitario portugués de 6 meses y en estos momentos se encuentran trabajando en Portugal, al tiempo que realizan la especialidad médica.

Por otro lado, en Portugal hay 40 médicos cubanos que vinieron a través de un acuerdo firmado en agosto de 2009 entre el Ministerio de Salud de Portugal y la Empresa de Servicios Médicos Cubanos. El contrato firmado por los respectivos ministerios de salud tiene una duración de tres años, que son renovables cada año. La mayoría de estos médicos cubanos trabajan en los centros de salud del Sur de Portugal (Alentejo y Algarve), que son las regiones más afectadas por la falta de médicos.

De todos modos, hay que señalar que una buena parte de los médicos uruguayos y cubanos que ejercen su labor en los servicios de salud portugueses, tanto públicos como privados, han emigrado de manera independiente y no a través de estos convenios bilaterales.

\section{CONSIDERACIONES FINALES}

España y Portugal forman parte de la dinámica de la migración internacional de los profesionales de la salud principalmente como países de destino, pero 
también como países de origen, en concreto España. Asimismo, las tendencias generales de esta migración observadas en el contexto mundial también se reflejan en ambos países: a lo largo de los últimos 20 años, Portugal ha experimentado un importante incremento en la llegada de estos profesionales de la salud extranjeros, fundamentalmente por su escasez estructural de personal sanitario; mientras que España, de manera más tardía, ha visto aumentar de manera significativa el número de inmigrantes profesionales de la salud en los últimos años. La tendencia a la mayor diversificación en los orígenes también se manifiesta en ambos países (aunque de manera más clara en Portugal) debido, en parte, al aumento de los flujos migratorios procedentes de Europa del Este. Efectivamente, se está produciendo un aumento importante de los movimientos migratorios de los profesionales de la salud procedentes de Europa del Este como consecuencia de la ampliación de la Unión Europea y, dentro de éste, Portugal y España se configuran como destinos de esta movilidad intraeuropea.

La importancia de los vínculos históricos y culturales se pone de manifiesto en la migración de los profesionales de la salud en Portugal procedentes de sus ex -colonias. Y de la misma forma, estos factores son evidentes en el flujo migratorio de estos profesionales en España oriundos de países de América Latina. Además, cabe añadir que estos respectivos flujos migratorios siguen los patrones de la migración en general en los dos países ibéricos.

El artículo también ha puesto de relieve la influencia de factores como las redes migratorias, la proximidad geográfica, cultural y lingüística, así como la combinación de algunos factores de atracción y expulsión relacionados con la dimensión profesional, para explicar la movilidad de los profesionales españoles a Portugal. Por su parte, se detecta la relevancia del factor político en la migración de médicos cubanos y uruguayos en Portugal, como resultado de políticas activas de reclutamiento a través de acuerdos bilaterales que tienen una enorme influencia en el desarrollo y condiciones en que se produce esta migración.

\section{BIBLIOGRAFÍA}

ADEL, Madelon den; BLAUW, Wim; DOBSON, Janet, HOESCH, Kirsten \& SALT, John (2004), "Recruitment and the migration of foreign workers in Health and Social Care", In Bommes, Michael; Hoesch, Kirsten; Hunger, Uwe; Kolg, Holger (ed.), Organisational Recruitment and Patterns of Migration. Interdependencies in an Integrating Europe, (IMIS-Beiträge, Special Issue 25), Osnabrück, pp. 201-230.

BAGANHA, Maria Ioannis e RIBEIRO, Joana Sousa (2007), "Imigração qualificada no sector da saúde-as oportunidades do mercado laboral português", In Dias, Sónia 
(org.), Revista Migrações-Número Temático Imigração e Saúde, $\mathrm{n}^{\circ}$ 1, Lisboa: ACIDI, pp. 53-78.

BUCHAN, J. y DOVLO, D. (2004), International recruitment of health workers to the UK: a report for DFID. London, Department for International Development, DFID Health Systems Resource Centre.

BUCHAN, J.; JOBANPUTRA, R y GOUGH, P. (2004), London calling? The international recruitment of health workers in the capital. London, King's Fund. Disponible en: http://www.kingsfund.org.uk/document.rm?id=114

CLARK, Paul F; STEWART, James B. \& CLARK, Darlene A. (2006), "The globalization of the labour market for health-care professionals", International Labour Review, 145 (1-2), pp.37-64.

COLEGIO DE MÉDICOS DE BARCELONA (2009), Informe Anual 2009, Barcelona, Colegio de Médicos de Barcelona.

GONZÁLEZ LÓPEZ-VALCARCEL, B. y BARBER, P. (2007), Oferta y necesidad de médicos especialistas en España (2006-2030). Las Palmas, Universidad Las Palmas Gran Canaria.

KLINE, Donna S. (2003), "Push and pull factors in international nurse migration", Journal of Nursing Scholarship, $\mathrm{n}^{\circ} 35$ (2), pp. 107-111.

LEAL, Carla Sofia Sales (2003), A migração de enfermeiros espanhóis e a gestão dos recursos humanos dos hospitais portugueses, Tesis de Máster, Lisboa, ISCTE.

LOWELL, B. Lindsay (2008), "Highly Skilled Migration", In IOM, World Migration 2008: Managing Labour Mobility in the Evolving Global Economy, Vol. 4 World Migration Report Series, pp. 51-76.

MASSEY, Douglas S., ARANGO, Joaquín; HUGO, G.; KOUAOUCI, A.; PELLEGRINO, A. y TAYLOR, E. (1998), Worlds in motion: understanding international migration and the end of the Millenium. Oxford, Clarendon Press.

MINISTERIO DA SAÚDE (2009), Recursos Humanos Estrangeiros no Ministerio da Saúde -Evolução 2001-2007-, Lisboa, Administração Central do Sistema de Saúde, Unidade Funcional de Estudos e Planeamento de Recursos Humanos.

OECD (2007), "Immigrant Health Workers in OECD countries in the broader context of highly skilled migration", International Migration Outlook, SOPEMI 2007. Disponible en http://www.oecd.org/dataoecd/22/32/41515701.pdf

OECD (2010), "International Migration of Health Workers. Improving international co-operation to address the global health workforce crisis", OECD Policy Brief February 2010. Disponible en: http://www.oecd.org/dataoecd/8/1/44783473.pdf RAY, Kristin Michelle; LOWELL, B. Lindsay \& SPENCER, Sara (2006), "International Health Worker mobility: causes, consequences and best practices", International Migration, Vol. 44 (2), pp. 181-203.

SASSEN, Saskia (1988), The mobility of labor and capital. Cambridge, University Press. SAYAD, Abdelmalek (1998), A imigração ou os paradoxos da Alteridade. São Paulo, Edusp. SIMOENS, S. y HURST, J. (2006), "The Supply of Physician Services in OECD Countries”. OECD Health Working Papers, n. 21. 
STEWART, James B.; CLARK, Darlene A. \& CLARK, Paul F. (2007), "Migration and recruitment of healthcare professionals: causes, consequences and Policy responses", Focus Migration, Policy Brief $\mathrm{n}^{\circ} 7$. Hamburg Institute of International Economics Disponible en: http://www.focus-migration.de/typo3_upload/groups/ 3/focus_Migration_Publikationen/Kurzdossiers/PB07_Health.pdf

VALLE, Luís; FARMHOUSE, Rosário; MARQUES, Vera (2008), "Reconhecimento de habilitações acadêmicas de médicos e de enfermeiros imigrantes", In Peixoto, João (org.), Revista Migrações-Número Temático Imigração e Mercado de Trabalho, $\mathrm{n}^{\circ}$ 2, Lisboa: ACIDI, pp. 171-176.

WHO (2006), "Working Together for Health", The World Health Report 2006. Disponible en:http://www.searo.who.int/LinkFiles/Making_Pregnancy_Safer_ WHR06_en.pdf 